\section{P88 (continued)}

side, topping); local/conventional sourcing. Trays were identifiable by grade; schools were categorized according to number of prior years of F2S; item frequency served as proxy for exposure. The percent of students reporting trying and liking selected items were incorporated from a Knowledge \& Attitudes survey. Analyses were performed using a Tobit model to assess within-category differences in cups of waste.

Results: Many factors indicated differences in waste overall, for FV separately, and/or across time. Cooked fruits were wasted less than raw, while cooked vegetables were wasted more than raw. Where identified, locally-sourced items were wasted more than conventional (0.1 vs 0.2 cups wasted, $\mathrm{p}<.0001)$. Salad bar items were wasted more than main menu items (0.01 cups, $\mathrm{p}<.0001)$. FV sides and toppings were wasted less than entrees. Item frequency did not influence waste amount. Finally, length of time in F2S decreased waste.

Conclusions and Implications: Some factors were significantly associated with elementary-aged children's waste of school lunch FV items. These factors may be helpful to consider in terms of menu and food service environment offerings and their implications for school meal program solvency.

Funding: Center for Disease Control and Prevention Additional Funding: USDA Hatch Grant

\section{P89 Super Tasters and Mighty Movers: Connecting Nutrition and Activity Messages From the School to the Home}

Alexandra Burdell, MPH, alexburdell@gmail.com, Colorado State University, Department FSHN, 1571

Campus Delivery, Fort Collins, CO 80523-1571;

T. Conlon, MS; A. Lopez, BS; A. D'Hooge, MS, RD;

L. Bellows, PhD, RD

Objective: To extend and reinforce messages from The Food Friends program with elementary-aged children in the classroom, cafeteria/gym, and home to sustain behavior changes made in preschool.

Target Audience: Students attending 2 elementary schools in rural Colorado participated in the 5 month program in both kindergarten and 1st grade.

Theory, Prior Research, Rationale: A 5-unit "booster" program, based on Social Cognitive Theory and Social Marketing principles, was developed with The Food Friends' characters and themes of "Super Tasters" and "Mighty Movers".

Description: Classroom-based lessons, and signage for the cafeteria and gym, were implemented in 34 classrooms ( $\sim 80$ students). A home-based component, The Super Taster and Mighty Mover Club, was developed and 5 monthly packages were mailed to 1 st grade study participants $(n=98)$ to promote school-based messages in the home environment. Mailings consisted of a child newsletter, a bilingual parent newsletter, and educational enhancer (e.g. spatula, jump rope, cookbook).
Evaluation: Child intercept interviews were conducted to determine if children could recall key program messages. A parent survey was sent home to gauge impressions of materials and understanding and incorporation of messages/materials in the home. Overall, the program was very well received by both children and parents. The majority (80\%) of children could articulate program messages without prompting (100\% with prompting). Parents (93\%) reported that materials encouraged their children to partake in targeted behaviors; $\sim 50 \%$ of families did the suggested activities/recipes and 20\% planned to in the future.

Conclusions and Implications: The efficacy of The Food Friends elementary component on behaviors will be evaluated as part of a larger longitudinal study.

Funding: USDA Grant \#2010-85215-20648

\section{P90 Development and Evaluation of a Textbook for Reducing Sugar Intake of Schoolchildren: Eat Smart Sweets}

KyungheaLee,PhD, khl@changwon.ac.kr, Changwon National University, Sarim-dong uichang-gu, Changwon, Gyeongnam, 641-773, Korea; T. Woo, PhD

Objective: This study was conducted to develop and evaluate a textbook for reducing sugar intake of 1st-3rd grade school children. Also, we tried to apply the more affecting factor for behavior motivation. Although the amount of sugar intake is still lower than Korean recommended dietary allowance, it would need to be treated as prevention.

Target Audience: 1st-3rd grade school children.

Theory, Prior Research, Rationale: The social cognitive theory was applied on this study. Prior to designing the textbook, the needs of the learner were analyzed. The textbook was designed based on analysis of previous studies. The study was approved by Institutional Review Board of Sungshin Women's University (swuirb2013028).

Description of Program Intervention: In prior survey, self-efficacy was the most important factor in self-control to refrain from sweets. Therefore, we designed the textbook focused on improving self-efficacy for consuming less sweet foods. Improving self-efficacy, education activities, such as the sensory exposure, peer's observation, experience for snack choice and positive feedback were chosen. The book consisted of three parts. The themes of the parts were 'Understanding the sweet preference', 'Behavioral assessment for the sweet food intake' and 'Eat less sweet foods'.

Evaluation: For evaluation of the education effect, we have conducted classes for 187 schoolchildren. After education, sweet food frequency $(\mathrm{p}<0.05)$, self-efficacy $(\mathrm{p}<0.001)$, outcome expectation $(\mathrm{p}<0.001)$ and nutrition knowledge $(\mathrm{p}<0.001)$ were significantly improved whereas attitude and environment were not changed. The score of education satisfaction was $3.84 \pm 0.72$ (total score-5).

Continued on page $S 143$ 
P90 (continued)

Conclusions and Implications: We implemented education using the textbook focused on increasing selfefficacy. The textbook improved not only self-efficacy but also sweet food frequency. The nutrition education using this textbook was contributed for reducing sugar intake in schoolchildren.

Funding: Ministry of Food and Drug Safety (13162MFDS160)

\section{P91 Turning Kids Into Food Detectives: The Pure Food Kids Workshop!}

Katherine Doughty, MS, IBCLC, Katie.wetzel@gmail.com, Flagship Foundation and University of Washington, 3116 164th Street SW, \#1102, Lynnwood, WA 98087;

K. Hyde; J. Saturay; C. E. Huebner, PhD, MPH, University of Washington

Objective: The purpose of the workshop is to develop food literacy skills in school-aged children by teaching them to become "food detectives".

Target Audience: The program was evaluated with a diverse sample of 4 th and 5 th graders in Washington State. Theory, Prior Research, Rationale: Gaining these skills is critical in a time of record rates of overweight and obesity. Childhood is the ideal time to intervene while health habits are being formed.

Description: During an interactive, 2.5 hour workshop children learn about food marketing, nutrition labels, ingredient lists, food additives and cooking skills. The goal is to increase awareness of the content of food choices.

Evaluation: A quiz highlighting each part of the workshop was administered pre and post workshop. An example question is, "Ingredients on a food label are listed: a.) Randomly in no special order b.) In order of least amount to greatest amount c.) In order of greatest amount to least amount d.) Don't know". Aggregated data from 103 classes, including 2,426 students, were analyzed. Paired sample t-tests showed highly significant improvements in students' responses to each question $(\mathrm{p}=0.001)$. Students also rated the degree to which they gained nutrition and cooking skills knowledge and their satisfaction with the workshop on scales of 1-10, with 10 being most favorable. The average knowledge-gained score was 8.2 and their satisfaction rating was 9.0 (of 10).

Conclusions and Implications: Students enjoyed this workshop while increasing their nutrition knowledge in each of the areas addressed.

Funding: Beecher's Flagship Foundation

\section{P92 Implementation of the Sprouts Growing Healthy Habits Curriculum in Preschool and Kindergarten Classrooms: Is it Feasible?}

Meghan Fisher, MA, mfisher2@illinois.edu, University of Illinois at Urbana-Champaign, 147 ECDL, $1005 \mathrm{~W}$.

Nevada Street, Urbana, IL 61801; B. Fiese, PhD
Objective: The objective of this pilot study is to analyze the overall feasibility of implementing a healthy habits curriculum in preschool and kindergarten classrooms.

Target Audience: This presentation targets administrators and teachers of preschool and kindergarten classrooms, as well as individuals interested in nutrition education for children.

Theory, Prior Research, Rationale: Teachers report low levels of nutrition knowledge due to minimal former education in these areas. Therefore there is a need to assist teachers in overcoming the barriers to teach healthy habits in their classrooms.

Description: This feasibility study explored barriers to and needs of implementing a healthy habits-focused curriculum, Sprouts: Growing Healthy Habits. The curriculum lessons were executed to 132 preschool and Kindergarten children by trained students. Head teachers observed the lessons. A one-time survey was distributed to the 6 head teachers at program completion.

Evaluation: All teachers viewed teaching healthy habits to this age group as important, and the majority viewed the lessons as age appropriate. Majority said they would be comfortable and that it was feasible to implement the lessons themselves. However, the majority identified the need of assistance from an outside organization due to financial reasons and time constraints.

Conclusions and Implications: Despite teachers' reported comfort levels in teaching this curriculum, 50\% of teachers said they did not have a curriculum that fulfills the needs of teaching healthy habits (the other half of teachers did not report if they did or did not have a comparable curriculum). Therefore, providing pre-prepared lessons and outside financial assistance for materials to implement healthy habits-focused curriculums is essential. Funding: Family Resiliency Center: Christopher Family Foundation Food and Family Program

\section{P93 A Gourd Smorgasbord Offers Second Grade Students Sensory Exposure to a Variety of Squash While Teaching Science}

Virginia Carraway-Stage,PhD, RD LDN, carrawaystagev@ ecu.edu, East Carolina University, E 1000 5th Street Rivers 266, College of Human Ecology, Greenville, NC 27834; J. Hammond, BA, Oakwood Elementary School; M. W. Duffrin, PhD, RD, LDN, East Carolina University

Objective: To share the layout and design of an activity called Gourd Smorgasbord, illustrate the activity alignment to Next Generation Science Standards, and discuss the implementation of the activity.

Target Audience: Second grade students.

Theory, Prior Research, Rationale: The Food, Math, and Science Teaching Enhancement Resource (FoodMASTER) Initiative uses food as a tool to teach mathematics and science. This project was a partnership with a teacher to enhance an existing thematic curriculum about pumpkins. 